\title{
National Institute of Arthritis and Musculoskeletal and Skin Diseases
}

National Cancer Institute

\section{Source}

National Cancer Institute. National Institute of Arthritis and Musculoskeletal and Skin

Diseases. NCl Thesaurus. Code C82605.

An institute within the National Institutes of Health that supports research into the causes, treatment, and prevention of arthritis and musculoskeletal and skin diseases, the training of basic and clinical scientists to carry out this research, and the dissemination of information on research progress in these diseases. 